\title{
Hint based Power Efficient Routing Protocol for MANETs
}

\author{
B.Manasa \\ Research Scholar \\ Department of Computer \\ Applications, Karunya University, \\ Coimbatore, India
}

\author{
P. Vijayalakshmi \\ Assistant Professor \\ Department of Computer \\ Applications, Karunya University, \\ Coimbatore, India
}

\begin{abstract}
Energy efficient hint based routing protocol (EEHP) in wireless networks is the problem of finding energy efficient shortest active path which also senses the node breakage before occurrence of disconnection and performs a handoff. In the existing Hint Based Routing Protocol (HBP), the nodes discover an active path to the destination exploiting a set of routing meta-information (called hints). The active paths based on hint computation is alone not sufficient for nodes in the ad hoc network for which there may be energy loss during packet transfer leading to disconnection. to avoid path loss a new energy efficient hint based routing approach outperforms the existing HBP protocol by inheriting the node handoff strategy into the HBP protocol which finds the active shortest path based on hint computation followed by route maintenance based on power of the nodes in the active route. The simulation results show that the proposed protocol performs well when a link failure occurrence is identified preventing the route from being broken, leading to energy efficient and better performance in the network.
\end{abstract}

\section{Keywords}

MANETs, HBP, hints, energy efficient, disconnection.

\section{INTRODUCTION}

Energy management in wireless networks is today's attractive research field, due to limited energy available in these wireless devices. Though the availability of energy is less, the wireless communication consumes much energy which is to be minimized. A suitable energy aware routing strategy [1] for wireless networks is to use those wireless nodes with good energy range and avoid those with low energy levels.

Routing protocols in ad hoc networking is classified into two types: (i) Table-driven (proactive), (ii) Source initiated on-demand (reactive) and (iii) Hybrid routing protocol. Proactive routing protocols always maintains a routing table causing much overhead but reactive protocols compute the route to the destination only on-demand and maintain the routing table for a limited period. Hybrid routing protocols are the combination of both proactive and reactive routing protocols working principle. In all the three categories of ad hoc routing protocols the design of the algorithm is in such a way that when a path fails due to link failure, and before a new path is established the packets in the network experiences large delays. The routing strategy utilizes the concept of Hint Based Routing Protocol [3] in finding the path to the destination based on the time vector. This research works active route trace back alone is not sufficient for effective routing in wireless networks. The energy levels of the nodes in the active path should also be considered for efficient routing without disconnection of network. A good energy-aware routing technique should balance two goals: choosing a shortest path with active nodes and maintaining the path with active nodes based on energy values. The first technique introduces a framework for packets forwarding in mobile environments, which exploits metainformation (in the form of hints) to direct a packet towards the general direction of the destination. The second technique (i.e.) node handoff can be categorized under route maintenance mode. Using the meta-information the active path to the destination is computed after which the energy levels of the nodes in the active path are to be monitored which is taken care of node handoff technique. This node handoff technique tries to find suitable node by again verifying the hint table followed by the verification of power values of the best hint node ((i.e.) the node with lower hint value). As we have incorporated our proposed energy efficient hint based protocol into the AODV [15] implementation and have run simulations to assess its impact on routing performance, we use the HELLO messages for periodic updation of tables used in this protocol.

\section{RELATED WORK}

Our interest is energy efficient routing among the nodes in the wireless ad hoc networks. [3] proposed a unicast protocol that finds an active path to the destination on-the-fly by computation called hints which are the meta-information of the nodes in the network that is used for finding the active route which is not sufficient for wireless networks. [13] proposed an On-Demand minimum energy routing for energy efficient routing in wireless ad hoc network where the routing protocol DSR uses cache for storing both route information and also energy information of the nodes in the route leading to little overhead when compared to normal on-demand protocols. [6], demonstrates that adding proactive route selection and maintenance to DSR and AODV significantly reduces the number of broken paths with a small increase in protocol overhead. [14] describes that the usage of nodes energy for sending packets decreases if the neighbor nodes are near off also multihop routes saves energy. As the mobility increases it is found that the minimum energy routing protocol performance degrades. [2], implemented a new broadcast algorithm called adjusted probabilistic flooding on the AODV, which reduces the overhead of route discovery process by increasing the rebroadcast probability of low density nodes and decreasing in high density nodes. This improves the saved broadcast upto $50 \%$ without affecting reachability under high mobility and density. [5] proposes an energy-aware routing mechanism which balances the energy consumption among the nodes of the network. [8] introduces a routing scheme that uses the feedback information carried in unicast packets and computes 
the path to the destination without using any extra overhead. Depending on feedback again a disadvantage as there may be loss of feedback during link breakage. [12] propose an extensible OnDemand power management framework for ad hoc networks that adapts to traffic loads where the nodes maintains soft-state timers that determine power management transitions which leads to reduction in energy consumption nearly to $50 \%$ when compared to a network without power management under long-lived cbr traffic. When mobility is high, intermediate nodes may remain in active mode longer than necessary, which results in reduced energy savings. [9] in two topology-unaware MAC protocols a finer energy consumption study is carried out in the sequel, focusing on the amount of energy consumed to successfully transmit a certain number of packets. But the increased number of transmissions (both successful and corrupted) under the probabilistic policy increases the power consumed per frame. [11] a new gossip algorithm is designed to direct the gossip process towards the destination node for path discovery, saving up to $80 \%$ of broadcast transmission compared to pure flooding while $60 \%$ of nodes have to process a requesting packet.[10] proposed a three-hop Horizon Pruning (THP) algorithm to compute Two-hop connected dominating set (TCDS) using only local topology information (i.e. 2-hop neighbor) which is ideal for the propagation of RREQ message in the route discovery process of on-demand routing protocols. THP is shown to be more efficient than all prior distributed broadcasting mechanisms, when a TCDS is preferred over connected dominating sets (CDS). But THP is not reliable when the topology changes frequently and there is a clear trade-off between reliability and efficiency. [4] proposed a bypass routing, local recovery protocol that aims to reduce the frequency of route request floods triggered by broken routes which when detected a node patches the affected route using local information, that is acquired on-demand, and there by bypasses the broken link. The bypass routing is performed after the broken link is detected leading to little overhead. [7] describes a localized protocol where each node requires only the knowledge of its distance to all neighbor nodes and distance between its neighboring nodes.

\section{PROBLEM DEFINITION AND PROPOSED SOLUTION}

In MANETs monitoring the nodes, reducing routing overhead and efficiently using the resources are the major concerns. As the nodes in these networks are mobile and have no fixed place, the reach ability information for that particular node must be available for all neighboring nodes.

We consider a network composed of $\mathrm{N}$ mobile nodes in which two nodes can be categorized as neighbors if they lie within one another's transmission range. Two neighbors can communicate according to the following primitives. A node i can issue:

a) send (pck, k) to send the packet pck to $\mathrm{k}^{\text {th }}$ node and thus knowing the result of the transmissions.

b) brcast (pck) to send the packet pck to the nodes within the transmission range.

A node can detect any other nodes movement i.e. in or out of its range. When the distance between two nodes say $i$ and $j$ increase (decrease) and it reaches $R$ at time $t$, we say that $i$ and $j$ lose their contact (come in contact) at time t. From the time when they came in contact until they lost the contact we can call them neighbors. This time interval is called contact time and the duration of the link between $\mathrm{i}$ and $\mathrm{j}$ denoted by $\tau_{\mathrm{ij}}$. Given a source $\mathrm{s}$, a destination $\mathrm{d}$ and a packet to be routed, defines two problems formally: i) Hint Based Shortest path computation:

Find a path $\mathrm{p}(\mathrm{s}, \mathrm{d})$ based on time vector $\mathrm{VH}_{\mathrm{i}}$ of every node $\mathrm{i}$. (i.e.) the nodes discover an active path to the destination exploiting a set of routing meta-information (called hints).

ii) Link Maintenance:

Find a path $\mathrm{p}(\mathrm{s}, \mathrm{d})$ based on time vector $\mathrm{VH}_{\mathrm{i}}$ such that the nodes participating during transmission of packets are within the minimum energy range.

\subsection{Algorithm Description}

Describing the algorithm in general terms, each node $i$ in the network is in charge of computing the hints $H_{i d}$ and power $p_{v l}$ for any possible destination node $d$. The hint $H_{i d}(t)$ computed [3] at time $t$ is zero if the $i$ and $d$ are neighbors at this time and it is infinity if they never came in contact before $t$. When a node $\mathrm{k}$ needs to forward a packet destined to $d$, it sends the packet to the neighbor $\mathrm{k}^{*}$ which provides the best hint and best power values among the neighbors that never forwarded the same packet before.

\subsection{Algorithm Details}

Here we try to describe the components of our proposed protocol namely Hint Computation [3], Node Handoff Computation, Hint and Power Values Gathering and Packet Forwarding that are implemented.

\subsubsection{Hint Computation [3]}

Each node i broadcast a heartbeat packet every $\Delta T_{b} s$ and uses the beacon received from the neighbors to manage a vector of time information, $V H_{i}$ which stores the relevant time information for other potential destinations. The entry for a destination $\mathrm{j}$, say $V H_{i}[j]$, stores: $V H_{i}[j] . t_{\text {start }}$, the time when the first heartbeat from $\mathrm{j}$ was detected; $V H_{i}[j] . t_{b r k}$, the time when the link with $\mathrm{j}$ was broken (the value is 0 if $\mathrm{j}$ is currently neighbor), $t_{\text {last }}$, the time of the last heartbeat received from $\mathrm{j}$; and $V H_{i}[j] . \tau$, the duration of the link with $j$. (i.e.) If hint value $=0$ then neighbor node and the destination nodes are neighbors, else we compute hint value [3] as:

$H i d=\left(V H i[j] . t_{\text {start }}-V H_{i}[j] . t_{b r k}\right) / V H_{i}[j] . \tau$

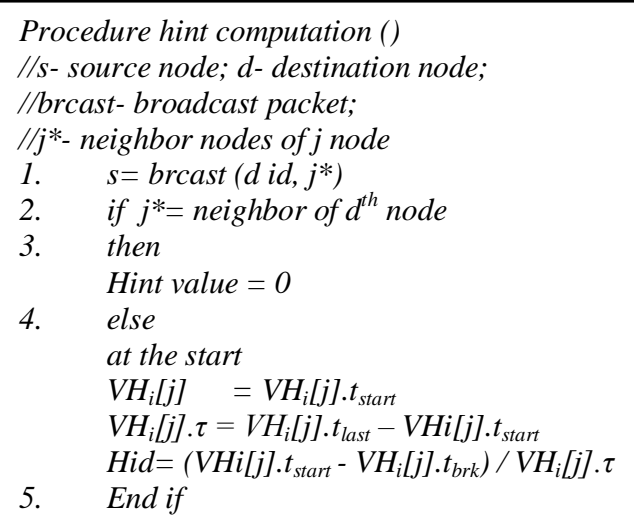

\subsubsection{Node Handoff Computation}

The decision of handoff is made by a node based on the signal strength of its neighbors with whom it forms part of an active route based on hints. Power information is maintained at each node in terms of the ratio of received power to the receive threshold power (HTTHRESH). If this ratio drops below the 
minimum value, then a node can no longer be heard. i.e. if the present nodes strength determined to be weak then we go for the node with a condition that received power or current power is greater than or equal to the HTTHRESH value or power difference of the nodes is equal to 0 .

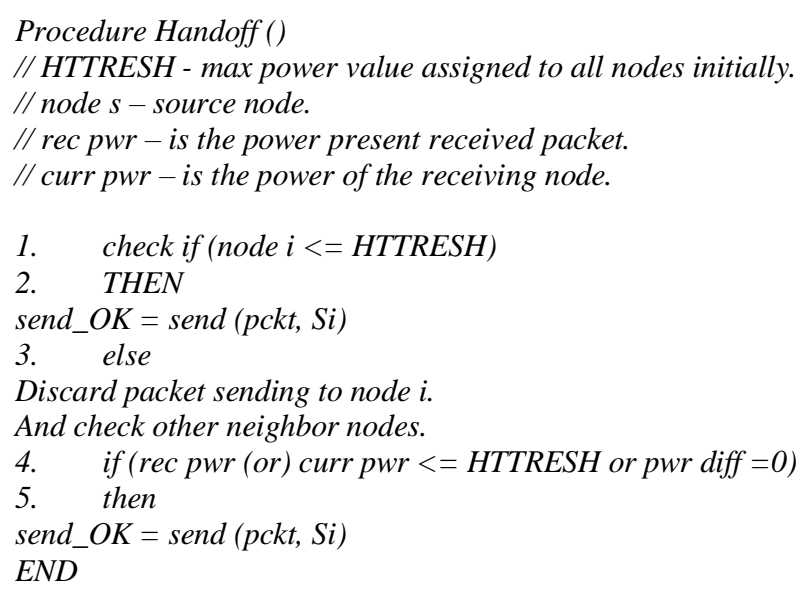

\subsubsection{Hint and Power Values Gathering}

Each node maintains a hint table, neighbor's power table and power difference table.

Hint Table [6]: It consists of (node id, hop count, next hop, gen, hint, dest id). In a $H_{i}$ of a node i namely ( $\mathrm{id}, \mathrm{h}, \mathrm{nh}, \mathrm{g}, \mathrm{h}$, dest) indicates that for the destination $d$ the hint $h$ has been received from the neighbor node $\mathrm{n}$ and that hint is generated by the node $\mathrm{g}$, which is hops away from node i. Hints dissemination is achieved by broadcasting control messages, which are carried by beacon packets.

Neighbor Power List Table (NPLT): Each node maintains an NPLT containing the last received signal for packets received from each neighbor. This table is updated each time when a packet is received and this happens atleast once every hello interval.

Power Difference Table: Each node also maintains power difference table. Here the difference in powers between a received packet and the entry in the NPLT for the last received packet from the neighbor is calculated and updated in the same way as NPLT and Hint table.

Since all these tables communication is carried out through hello packets, these hello packets are sent at regular periodic interval of $1 \mathrm{sec}$.

\footnotetext{
Procedure update ()

$/ / \Delta T_{b} S-$ Heart beat

I/n id-node id; hc-hop count; nh-next hop;

//g-neighbor node; h-hint; d id-dest id;

//pwr-power value

1. for every $\Delta T_{b} S d o$

2. Delete stale entries from hint table and power table.

3. $\quad$ brcast (dest id, $k^{*}$ )

4. Update Hint table ( $\mathrm{id}, \mathrm{hc}, \mathrm{nh}, \mathrm{g}, \mathrm{h}, \mathrm{d}$ id).

5. Update power table ( $n$ id, pwr,time, diff)

6. Update NPLT ( $n$ id, pwr diff)

7. End
}

\subsubsection{Packet Forwarding}

On receiving a packet destined to $\mathrm{d}$, a node $\mathrm{i}$ forwards it to the neighbor from which the best(lowest) hint and best(greater or good range) power value has been received and such that it did not see the packet before. If there are no such nodes existing then the packets are just discarded without selecting any node for transmission. Thus the above procedure is repeated at each packet forwarding step. This algorithm is very clearly resilient to topological changes.

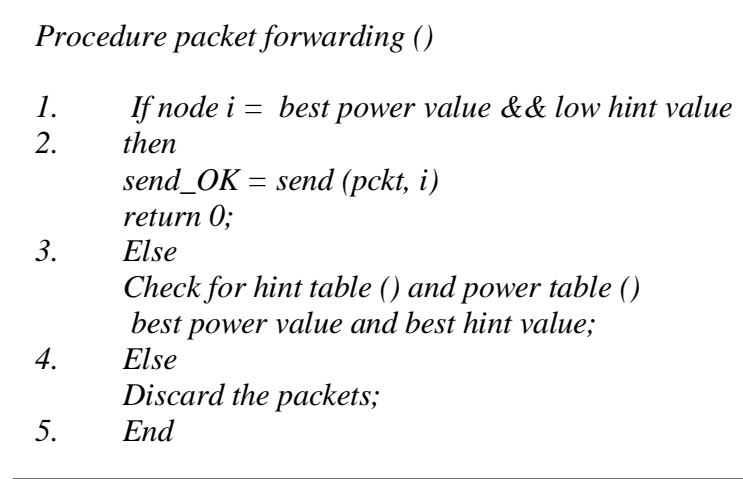

\section{PERFORMANCE ANALYSIS}

We now discuss the performance of our EEHP on general topologies. We use NS2 as our simulation tool [16], [17].

\subsection{Topologies used:}

We use a topology which is similar to that used for HBP protocol for comparison. The topography used is of $500 \times 500$ with 25,50 , 75,100 nodes. We increase the number of nodes while keeping the total area constant, thus increasing the density. The transmission range is $250 \mathrm{mts}$.

\subsection{Mobility:}

Nodes can move to a region of say $\mathrm{E} \mathrm{kms}$ according to the TwoRayGround mobility model with zero pause time. At the beginning of the simulation, the nodes are placed randomly in the region. Each node then selects a new point and travels towards it at a constant speed as defined in the simulation parameters in the TCL script. The speed of the nodes is given as $20 \mathrm{~m} / \mathrm{s}$.

\subsection{Traffic pattern:}

Packets are generated by CBR traffic pattern sources. Here a source always sends packets to the destination.

\subsection{Energy consumption:}

We compare two protocols HBP and EEHP based on energy consumption and packet delivery without link breakage inbetween the communication. We compare the two protocols based on the number of nodes by the energy consumption.

\subsection{Control Overhead:}

The protocols HBP and EEHP when compared based on control overhead we found that HBP protocol incurs little more overhead than our proposed protocol. 


\subsection{End-End delay:}

The end to end packet delivery is compared. There may cause delay during the node handoff. In HBP protocol the delay is caused much during the route discovery and retransmission. But in our proposed protocol the delay is caused during the node handoff only. As the number of nodes increases there is a slight increase in our proposed protocol.

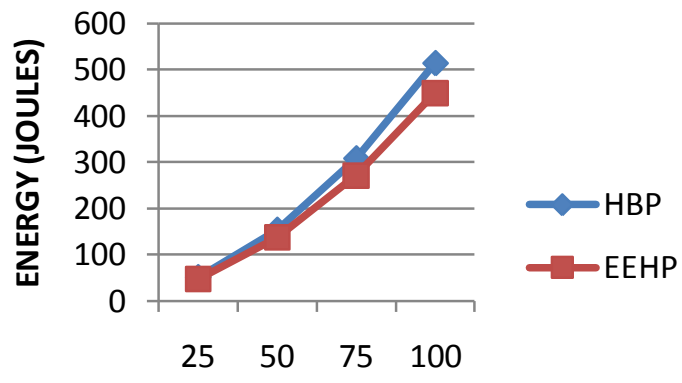

No. of Nodes

Figure 1: No. of Nodes vs. Energy (joules)

Table 1. The readings of two protocols based on energy consumption

\begin{tabular}{|l|l|l|l|l|}
\hline $\begin{array}{l}\text { No. of } \\
\text { Nodes }\end{array}$ & 25 & 50 & 75 & 100 \\
\hline $\begin{array}{l}\text { HBP } \\
\text { (in } \\
\text { Joules) }\end{array}$ & 51.0632 & 153.63 & 308.161 & 514.24 \\
\hline $\begin{array}{l}\text { EEHP } \\
\text { (in } \\
\text { Joules) }\end{array}$ & 46.94 & 137.04 & 271.36 & 449.24 \\
\hline
\end{tabular}

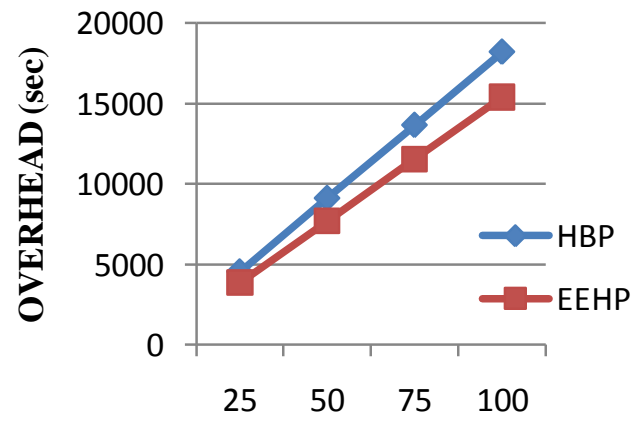

No. of nodes

Figure 2: No. of Nodes vs. Control Overhead (sec)
Table 2. The readings of two protocols based on control overhead

\begin{tabular}{|l|l|l|l|l|}
\hline $\begin{array}{l}\text { No. of } \\
\text { Nodes }\end{array}$ & 25 & 50 & 75 & 100 \\
\hline $\begin{array}{l}\text { HBP } \\
\text { (in sec.) }\end{array}$ & 4548 & 9119 & 13668 & 18222 \\
\hline $\begin{array}{l}\text { EEHP } \\
\text { (in sec.) }\end{array}$ & 3860 & 7707 & 11563 & 15400 \\
\hline
\end{tabular}

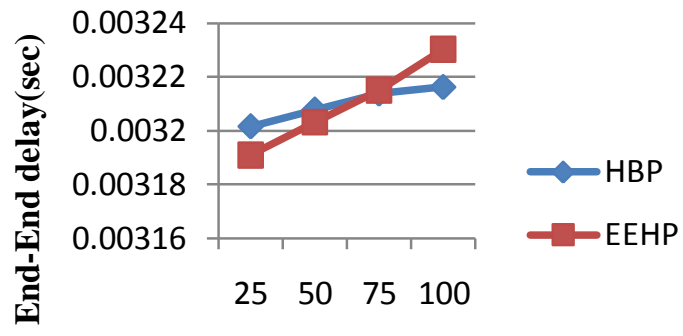

No. of nodes

Figure 3: No. of Nodes vs. End-to End delay (sec)

Table 3. The readings of two protocols based on end to end delay

\begin{tabular}{|l|l|l|l|l|}
\hline $\begin{array}{l}\text { No. of } \\
\text { Nodes }\end{array}$ & 25 & 50 & 75 & 100 \\
\hline $\begin{array}{l}\text { HBP } \\
\text { (in sec.) }\end{array}$ & 0.003201 & 0.003207824 & 0.003213 & 0.0032162 \\
\hline $\begin{array}{l}\text { EEHP } \\
\text { (in sec.) }\end{array}$ & 0.003190 & 0.00320313 & 0.003215 & 0.003230 \\
\hline
\end{tabular}

\section{CONCLUSION AND FUTURE WORK}

This paper proposed a hint based energy efficient protocol for unicast packet delivery in mobile ad hoc networks. The proposed hint based power efficient protocol has shown better performance based on energy consumption. Instead of using classical path computation, the path for forwarding packets is chosen by hint and power value computation within a small number of hops $\mathrm{L}$. In future this hint based power efficient protocol concept can be implemented on other multicast routing protocols and also check the performance for maximum number of hops. And also we try to implement in hybrid routing protocols with minor changes. 


\section{REFERENCE}

[1] Aravind B Mohanoor, Sridhar Radhakrishnan, Venkatesh Sarangan,"Online energy aware routing in wireless networks", Ad Hoc Networks 7 (2009) 918-931.

[2] Bani-Yassein, M. and Ould-Khaoua, M. and Mackenzie, L.M. and Papanastasiou, S. and Jamal, A. "Improving route discovery in ondemand routing protocols using local topology information in MANETs." In, Proceedings of the ACM International Workshop on Performance Monitoring, Measurement, and Evaluation of Heterogenous Wireless and Wired Networks, 2 October 2006.

[3] R. Beraldi, L. Querzoni, R. Baldoni, "A hint-based probabilistic protocol for unicast communications in MANETs", Ad Hoc Networks 4 (5) (2006) 547-566.

[4] Cigdem Sengul, Robin Kravets, 'Bypass routing: An on-demand local recovery protocol for ad hoc networks “,Ad Hoc Networks 4 (2006) 380-397.

[5] Daniel de O. Cunha Lu'is Henrique M. K. Costa Otto Carlos M. B. Duarte, "An Energy-Aware Routing Mechanism for Mobile Ad Hoc Networks" This work has been supported by CNPq, CAPES, COFECUB, and FAPERJ, 2003

[6] T. Goff, N.B. Abu-Ghazaleh, D.S. Phatak and R.Kahvecioglu, "Preemptive Routing in Ad Hoc Networks," Proc. of ACM MobiCom, 2001

[7] Julien Cartigny, David Simplot, and Ivan Stojmenovic,'Localized Minimum-Energy Broadcasting In Ad-Hoc Networks", Ad Hoc Networks 4 (2006) 380-397.

[8] Keyvan Kashkouli Nejad, Shawish Ahmed, Xiohong Jiang, Susumu Horiguchi." Probabilistic proactive routing with active route trace-back for MANETs", Ad Hoc Networks 8 (2010) 640-653.
[9] Konstantinos Oikonomou , Ioannis Stavrakakis, "Energy considerations for topology-unaware TDMA MAC protocols", Ad Hoc Networks 4 (2006) 359-379.

[10] Marco Aure lio Spohn ， J.J. Garcia-Luna-Aceves, "Improving route discovery in on-demand routing protocols using two-hop connected dominating sets", Ad Hoc Networks 4 (2006) 509-531.

[11] Roberto Beraldi,"The polarized gossip protocol for path discovery in MANETs", Ad Hoc Networks 6 (2008) 79-91

[12] Rong Zheng, Robin Kravets, "On-demand power management for ad hoc networks", Ad Hoc Networks 3 (2005) 51-68

[13] Sheetalkumar Doshi, Shweta Bhandare, Timothy X Brown, "An On-demand Minimum Energy Routing Protocol for a Wireless Ad Hoc Network, Mobile Computing and Communications Review", Ad Hoc Networks July 2002 Volume 6, Number 3 .

[14] Sheetalkumar Doshi, Timothy X Brown, "Minimum Energy Routing Schemes for a Wireless Ad Hoc Network", IEEE INFOCOM 2002.

[15] Vikas Singla, Parveen Kakkar,"Traffic Pattern based performance comparision of Reactive and Proactive protocols of Mobile Ad-hoc Networks", International Journal of Computer Applications, 2010. 
Reader Notes: 